QUARTERLY OF APPLIED MATHEMATICS

VOLUME LXIX, NUMBER 4

DECEMBER 2011, PAGES 677-690

S $0033-569 X(2011) 01258-9$

Article electronically published on July 7,2011

\title{
MULTIPLE SCALES ANALYSIS OF WATER HAMMER ATTENUATION
}

\author{
$\mathrm{BY}$ \\ S. Y. HAN (Worley-Parsons Canada, 400-10201 Southport Rd. SW, Calgary, Alberta, Canada, T2W \\ $4 \times 9)$, \\ D. HANSEN (Department of Civil and Resource Engineering, Dalhousie University, 1360 Barrington \\ St., Halifax, NS, Canada, B3J 1Z1), \\ AND \\ G. KEMBER (Department of Engineering Mathematics, Dalhousie University, 1340 Barrington St., \\ Halifax, NS, Canada, B3J 1 Y9)
}

\begin{abstract}
A multiple scales analysis is used to construct a uniformly accurate approximation to water hammer pressure wave attenuation that is initiated by a sudden valve opening. The method of analysis is well suited to the study of a water hammer that possesses several time scales and is applied to a mild generalization of the classical equations. It should prove useful for finding attenuation curves when effects such as unsteady friction and fluid-structure interaction are added. The analytical results are numerically verified using the method of characteristics.
\end{abstract}

1. Introduction. The nonlinearity of the classical water hammer model and its various generalizations has led to the development of increasingly sophisticated numerical and semi-analytical methods of analysis that have proven useful in engineering applications. However, it remains of interest to find uniformly accurate approximations whenever possible that are not simply ad hoc. The development of a multiple-scale decomposition of the water hammer equations, one that may be capable of generalization to various boundary and initial conditions and to features such as unsteady friction, is the focus of this paper.

Numerical and semi-analytic studies have appeared for a wide range of water hammer problems. A semi-analytic solution was developed in [17] based on the use of a wavetracking algorithm to describe hydraulic transients in thin-walled conduits. The analysis of a limited number of 2-D unsteady velocity profiles is in [7], where flow stability is

Received March 2, 2010.

2000 Mathematics Subject Classification. Primary 76M99; Secondary 76M45.

E-mail address: Sang-Yoon.Han@WorleyParsons.com

E-mail address: David.Hansen@dal.ca

E-mail address: Guy.Kember@dal.ca

(C) 2011 Brown University

Reverts to public domain 28 years from publication 
described with respect to the 'spectrum' of turbulence at certain times and locations during the water hammer event. Other semi-analytic solutions include Adomian decomposition, which was applied in [1] to the problem of shock-wave propagation through an unconfined gas. Standard eigenfunction methods have also been used, and in [16] this approach was applied to the pressure-wave transmission through a conduit between two pressure-vessels. The method of characteristics has been useful to numerically investigate the effects of including such specialized aspects as cavitation, column separation, and leakage/blockage [4, 5]. It was found that Poisson-coupling, a very specific type of fluid-structure interaction, can lead to a 'beat' phenomenon in certain cases. The efficiency and accuracy of the outcomes of modelling hydraulic transients was compared in [11] using a finite-volume Godunov shock-capturing scheme and a traditional fixed-grid method of characteristics approach for both one- and two-phase flows. Good agreement between observations and experimental results was found. Wavelet decomposition has also been utilized in a standard Galerkin numerical scheme to solve the water hammer equations, and the results compared well with those found via method of characteristics [13.

In this paper, a generic multiple-scales analytical approach is developed for the classical water hammer equations. The method generalizes ad hoc results [10] through the application of a structured approach, multiple-scales, that is well suited to the construction of uniformly accurate approximations to equations characterized by the presence of several time scales. The structured approach followed here may also prove useful to analyze generalizations of the classical equations to water hammer models presented in [14] and to continue on from detailed discussions of time scales in unsteady flows that appear in [2] and [3]. Standard assumptions made here include a constant pipe-wall thickness, pipe supports that supply longitudinal restraint, and the presence of frequent axial expansion joints. The results found here are for low Mach number, linearly elastic conditions in which there is no cavitation, column separation, leakage, or blockage within the non-tapered conduit. Darcy-Weisbach estimates of friction are assumed to apply and other effects, including unsteady friction, are neglected. The above assumptions are common and the numerical analyses of such cases have been used in various engineering applications [18.

The paper is organized as follows: (i) the water hammer equations and nomenclature are given (Section 2), (ii) the problem is rescaled and dimensionless parameter sizes are found (Section 3), (iii) a multiple-scales decomposition is used to describe attenuation of pressure-wave amplitude for a water hammer initiated by valve opening. The analytical

results are verified by comparison with the same outcomes found via the method of characteristics (Section 44).

\section{Water hammer equations.}

2.1. Quadratic head loss formulation. Figure 1 is a schematic of the Moody diagram 12 governing steady flow in pressure conduits. It is essentially a summary of semiempirical results, obtained by others, from experiments done on pipes of different interior roughnesses. The Moody diagram was originally designed to allow for the graphical determination of such things as the friction-induced head loss associated with a given 


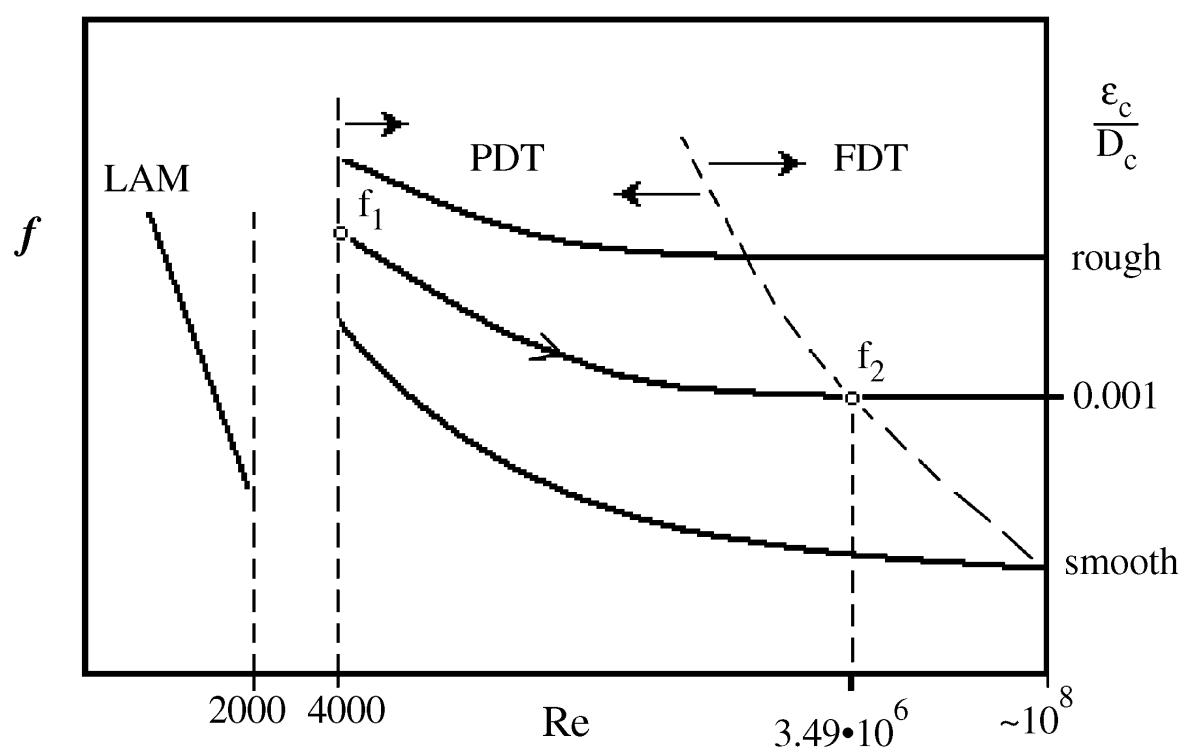

FIG. 1. Schematic representation of the Moody diagram governing steady flow in pressure conduits. It illustrates three flow regimes: (i) laminar (LAM), (ii) partially developed turbulence (PDT), and (iii) fully developed turbulence (FDT). Parameters $r$ and $s$ in equation (5) describe PDT flow between the points labelled $f_{1}$ and $f_{2}$. The arrow indicates the path during flow establishment. Note that the left-hand ordinate and the abcissa of the Moody diagram use log scales.

flow rate. The Moody diagram in Figure 1 also usefully illustrates some important phenomenology by visually separating the flow into three regimes. Its left-hand ordinate is the Darcy-Weisbach friction factor; an historical development is in [8], defined as

$$
f=\frac{(\Delta h / L) D_{c}}{v_{\infty}^{2} /(2 g)},
$$

where $v_{\infty}$ is the steady flow velocity, $\Delta h$ is the external head applied across the ends of the pipe of length $L$ and diameter $D_{c}$, and $g$ is the acceleration due to gravity. The Reynolds Number is given by

$$
R e=\frac{v_{\infty} D_{c}}{\nu}
$$

where $\nu$ is the kinematic viscosity.

A given conduit has a relative roughness $\epsilon_{c} / D_{c}$, where the absolute roughness $\epsilon_{c}$ is dependent upon the nature and condition of the material that makes up the inner wall of the pipe. The steady rate of flow through a given pipe is a point located on a given curve within the Moody diagram and its location is dependent, for example, on the external head applied across the ends of the pipe. A single 'Moody PDT curve' such as the one passing through $f_{1}$ and $f_{2}$, associated with a given conduit, may be described by

$$
f=\frac{M_{1}}{R e}+M_{2}
$$


where $M_{1}$ and $M_{2}$ are empirical quantities that are unique to a relative roughness $\epsilon_{c} / D$ of interest. Elimination of $f$ and $R e$ in equations (11), (2), and (3) gives

$$
\Delta h=M_{1} \frac{\nu}{g} \frac{L}{D^{2}} v+M_{2} \frac{L}{g D} v^{2},
$$

which may be represented by

$$
\Delta h=r v+s v^{2},
$$

a form first proposed in [6]. The values of $r$ and $s[9$ are conveniently inferred using regression analysis of a given Moody curve.

2.2. Momentum equation. The standard momentum equation is generalized here to include the quadratic head loss form of (5):

$$
g h_{x}+v v_{x}+v_{t^{\prime}}+\frac{g}{L}\left(r v+s v^{2}\right)=0 .
$$

The partial derivatives are conveniently written as subscripts and the quadratic head loss component $|v| v$ is written as $v^{2}$ since the flow is assumed to be free of reversals, thus ensuring that the shear stress will always oppose the motion [18. Flow reversals are not considered herein, but may be included by a piecewise analysis of the problem where the flow is in one direction, forward or backward, within each portion of the problem analyzed. Instead, the emphasis is placed on the presentation of a generic asymptotic approach which, to the best of the authors' knowledge, has only been considered before in an ad hoc fashion [10.

2.3. Continuity equation. The continuity equation used here is

$$
-\frac{\partial A \rho v}{\partial x}=\frac{\partial \rho A}{\partial t^{\prime}}
$$

with velocity $v$ and conduit cross-sectional area $A$. Expanding (7) and noting that the total derivative is $(v \partial / \partial x+\partial / \partial t)=d / d t^{\prime}$ yields

$$
\frac{1}{\rho} \frac{d \rho}{d t^{\prime}}+\frac{1}{A} \frac{d A}{d t^{\prime}}+\frac{\partial v}{\partial x}=0 .
$$

The continuity equation (8) is closed by relating changes in fluid density and in pipe elasticity to pressure. Respectively, $1 / \rho d \rho / d t^{\prime}=1 / E_{v} d p / d t^{\prime}$ [18, where $E_{v}$ is the volumetric bulk modulus of water, and $1 / A d A / d t^{\prime}=\eta D /(w E) d p / d t^{\prime}$, 18, where Young's modulus of the pipe material is $E$, pipe wall thickness is $w$ [18, and $\eta$ is the pipe-supportcondition coefficient. In [18, Poisson's ratio and the application of other longitudinal and/or radial restraint conditions can be used to compute appropriate values of $\eta$, which typically vary between about 0.75 and unity. A choice of $\eta=1$ is made here that implies zero axial stresses so that the occurrence of strain is confined to the radial direction (full details are in [18). Pipe slope relates to pressure as $p=\rho g(h-z)$ with $\partial z / \partial t^{\prime}=0$ and $\partial z / \partial x=\sin (\theta)$. The celerity is

$$
c_{p}=\sqrt{\frac{K}{\rho}},
$$

where

$$
K=\frac{E_{v}}{1+\eta \frac{E_{v}}{E} \frac{D}{w}}
$$




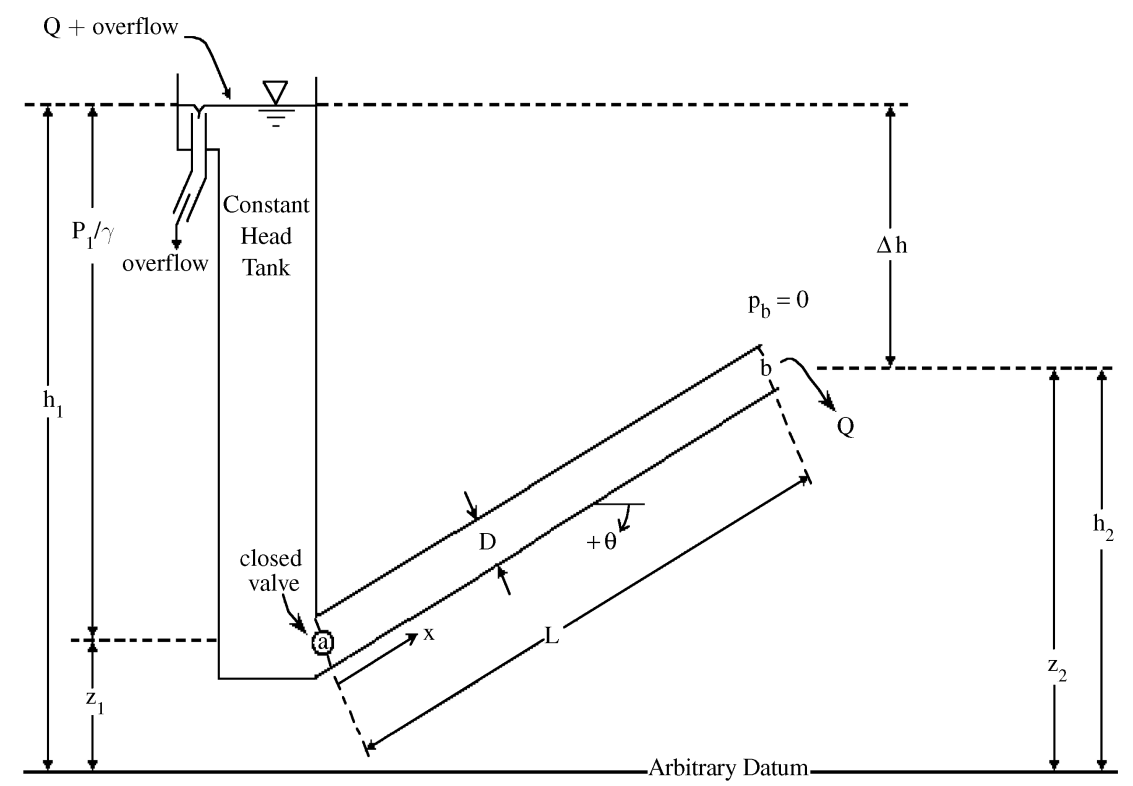

FiG. 2. Schematic of physical setup before $t^{\prime}=0$. Despite the presence of hydrostatic pressure along the conduit length before $t^{\prime}=0$, the conduit diameter $D_{c}$ is assumed to be single-valued.

from which the final form of the continuity equation is

$$
\frac{c_{p}^{2}}{g} v_{x}+v h_{x}+h_{t^{\prime}}+v \sin (\theta)=0 .
$$

Once again, the derivatives have been written as subscripts for convenience.

The water hammer is analyzed for the case of an instantaneous valve-opening, where the initial velocity is zero and the initial head is constant after which the boundary conditions $x=0, h=h_{1}, x=L, h=h_{2}$ are suddenly imposed, causing the water hammer. These conditions lead to a water hammer that repeatedly traverses the pipe, for example, in a flow establishment initiated by a controlled centrifugal pump delivering a constant head [15. In contrast, the water hammer initiated in the more familiar physical setup considered here in Figures 2 and 3 could only traverse the pipe once since it would dissipate after returning to and entering the tank.

3. Dimensional analysis. The dimensional momentum (6) and continuity (11) equations are rescaled for analysis. The head $h$ and velocity $v$ are rescaled using the difference in applied head $h_{12}=h_{1}-h_{2}$ and steady velocity $v_{\infty}$, respectively, giving $H=\left(h-h_{2}\right) / h_{12}$ and $V=v / v_{\infty}$. The length scale is taken to be the conduit length $L$ so that $X=x / L$. The choice of time scaling for this problem is chosen to balance the $h_{x}$ and $v_{t^{\prime}}$ in the momentum equation, which requires that

$$
T=\frac{L v_{\infty}}{g h_{12}}
$$




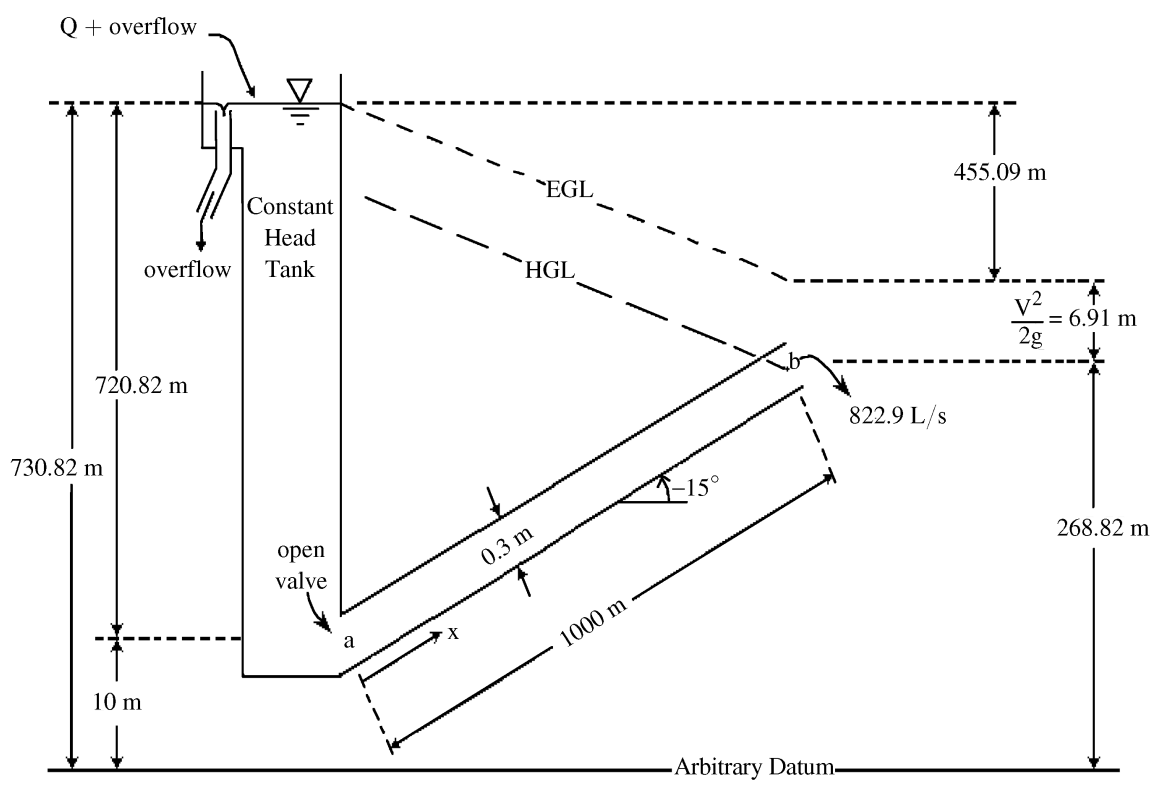

FIG. 3. Schematic of physical setup at steady state. Entrance losses at point "a" are neglected. (HGL = hydraulic grade line, EGL $=$ energy grade line.)

where dimensionless time is $\tau=t^{\prime} / T$. The time scale $T$ is equal to the inertial time scale, $v_{\infty} / g$, scaled by the hydraulic gradient $h_{12} / L$, and is the time scale over which the water hammer wave attenuates (Section 4.2). Note that, in the case of flow stoppage, $v_{\infty}$ is replaced with a representative value of the flow before the stoppage.

The three dimensionless parameters, in order of their appearance in the momentum equation, are

$$
C_{1}=\frac{v_{\infty}^{2}}{g h_{12}}, C_{2}=\frac{r v_{\infty}}{h_{12}}, C_{3}=\frac{s v_{\infty}^{2}}{h_{12}} .
$$

Similarly, those from the continuity equation are

$$
C_{4}=\left(\frac{g h_{12}}{c_{p} v_{\infty}}\right)^{2}, C_{5}=\frac{g L \sin \theta}{c_{p}^{2}} .
$$

The rescaled momentum and continuity equations are

$$
\begin{array}{r}
H_{X}+C_{1} V V_{X}+V_{\tau}+C_{2} V+C_{3} V^{2}=0, \\
V_{X}+C_{1} C_{4} V H_{X}+C_{4} H_{\tau}+C_{5} V=0
\end{array}
$$

with rescaled head, boundary, and initial conditions

$$
\begin{aligned}
& X=0, H=1 \\
& X=1, \quad H=0
\end{aligned}
$$

with zero initial conditions, i.e. $\tau=0, H=0$ and $\tau=0, V=0$.

In the momentum and continuity equations in (15), the dimensionless parameters represent the following: 
- The 'Advection Effect' is represented by $C_{1}$ and including density, $C_{1}=$ $\left(\rho v_{\infty}^{2}\right) /\left(\rho g h_{12}\right)$, we have $C_{1}$ as the ratio of a velocity head to a hydraulic head.

- The 'Viscous Effect' is seen in $C_{2}$. The parameter $r$ has units of seconds, so that $C_{2}$ is a kinematic 'viscous head' relative to the applied hydraulic head $h_{12}$.

- The 'Inertial Effect' is contained in $C_{3}$ and is increasingly dominant as the level of turbulence increases, since it represents the ratio of the equilibrium inertial head to the applied hydraulic head difference.

- 'Advective head' is represented in $C_{1} C_{4}$. If this term is recast as $\left[\rho g h_{12} /\left(\rho c_{p}^{2}\right)\right]$, then we have the ratio of hydraulic head difference (expressed as a pressure) to the pressure from the propagating wave.

- A 'Local' velocity is represented by $C_{4}$. If we introduce the density, then $C_{4}=$ $\left[\left(\rho g h_{12}\right) /\left(\rho c_{p} v_{\infty}\right)\right]^{2}$ and $\sqrt{C_{4}}$ is the ratio of the applied hydraulic head difference to the 'local' pressure head.

- 'Elevation head' appears in $C_{5}$ and this parameter shows the effect of the pipe slope elevation head in relation to the head from the propagating wave, a celerity head.

The dimensionless parameters from the momentum equation, $C_{1}, C_{2}$, and $C_{3}$, are treated as $O(1)$. The convective term $V V_{X}$ is typically neglected (e.g. as reviewed in (4) but is retained here because $C_{1}$ is $O(1)$; this is also true for the steady velocity, $v_{\infty}^{2}=O\left(g h_{12}\right)$, considered in the water main application in Section 4.3, The continuity parameters $C_{1} C_{4}, C_{4}$, and $C_{5}$ are presumed to be small and inversely proportional to the square of the celerity $c_{p} \gg 1$, and $C_{1}$ is order one. For convenience, these parameters are defined in the analysis as the equally contributing parameters $C_{1} C_{4}=C_{1} c_{4} \epsilon^{2}, C_{4}=c_{4} \epsilon^{2}$ and $C_{5}=c_{5} \epsilon^{2}$ with $C_{1}, c_{4}$, and $c_{5}$ being of order one. Once again, although the convective term $C_{1} c_{4} \epsilon^{2}$ is typically neglected, as in [4, it is retained here since it may have a comparable magnitude to $c_{4} \epsilon^{2}$ and $c_{5} \epsilon^{2}$. A natural choice for the artificial scaling constant $\epsilon$ used here is $\epsilon^{2}=O\left(1 / c_{p}\right)$. The final form of the momentum/continuity pair is then

$$
\begin{aligned}
H_{X}+C_{1} V V_{X}+V_{\tau}+C_{2} V+C_{3} V^{2} & =0, \\
V_{X}+C_{1} c_{4} \epsilon^{2} V H_{X}+c_{4} \epsilon^{2} H_{\tau}+c_{5} \epsilon^{2} V & =0 .
\end{aligned}
$$

4. Multiple scales. The gross functional behaviour of the typical water hammer is that of a fast wave superimposed on a slowly varying trend where the former appears to attenuate at the same timescale as the trend evolution. This behaviour is quantified in the following three sections: (i) the attenuating wave dimensionless timescale and scale of variation of the velocity are found, (ii) the slowly evolving trend (not always visible because water hammer dominates) is evaluated, and (iii) the full multiple-scale analysis and numerical comparison are completed.

4.1. Attenuating wave. The water hammer wave requires a balance between the dominant terms $H_{X}$ and $V_{\tau}$ in the momentum equation (18) and $V_{X}, \epsilon^{2} c_{4} H_{\tau}$ in the continuity equation (19) and this leads to the first approximation

$$
\begin{aligned}
H_{X}+V_{\tau} & =0, \\
V_{X}+c_{4} \epsilon^{2} H_{\tau} & =0,
\end{aligned}
$$


where friction and convection are neglected as lower-order phenomena. The mathematical justification for the dominance of the terms in (20) and over other terms dropped into the governing equations (18) and equation (19) will be apparent shortly.

The balance of terms in the reduced form (20) is established by assuming a unidirectional wave where $H=a f(X-b \tau)$ and $V=c f(X-b \tau)$, where $a$ and $c$ are magnitude scales, independent of the wave direction. The functional dependence of $H$ and $V$ on $X-b \tau$ is the same since they share the wave speed and period while the constants $b$ and $c$ involve the parameter $\epsilon$ with $a$ equal to order one. Substituting the assumed form of head and velocity into the reduced set yields

$$
\begin{aligned}
a-b c & =0, \\
c-c_{4} a b \epsilon^{2} & =0 .
\end{aligned}
$$

Eliminating $a=b c$ gives $c\left(1-c_{4} b^{2} \epsilon^{2}\right)=0$, which fixes $b=1 /\left(\epsilon \sqrt{c_{4}}\right)$ and $a=c /\left(\epsilon \sqrt{c_{4}}\right)$. We choose, without loss of generality, $a=1$ from which $c=\epsilon \sqrt{c_{4}}$. The resulting head and velocity are then $H=f\left(X-\tau /\left(\epsilon \sqrt{c_{4}}\right)\right)$ and $V=\epsilon \sqrt{c_{4}} g\left(X-\tau /\left(\epsilon \sqrt{c_{4}}\right)\right)$. Both of these functions share the short dimensionless time scale $t=\tau / \epsilon$, where $\tau=O\left(\epsilon \sqrt{c_{4}}\right)$. In other words, the wave is of the form $H=f\left(X-t / \sqrt{c_{4}}\right)$ and $V=\epsilon \sqrt{c_{4}} g\left(X-t / \sqrt{c_{4}}\right)$, where $t$ is $O(1)$ or $\tau$ is $O(\epsilon)$. The mathematical basis for the reduction in equation (20) is now apparent since: (i) $H_{X}$ and $V_{\tau}$ are $O(1)$ in equation (18) while other terms are $O(\epsilon)$ and below, and (ii) $V_{X}$ and $\epsilon^{2} H_{\tau}$ are $O(\epsilon)$ with the remaining terms being $O\left(\epsilon^{3}\right)$.

The reduced set in equation (20) is a first approximation to the attenuating wave represented in the full equations (18) and (19). Our analysis of this section shows that the head and velocity are dependent upon two timescales: (i) a wave oscillation timescale $t$ that corresponds to $\tau=O(\epsilon)$ and (ii) a wave attenuation timescale at $\tau=O(1)$. Thus, the head and velocity represented in the full equations (18) and (19) may be written as $H=H(X, t, \tau)$ and $V=\epsilon \sqrt{c_{4}} V(X, t, \tau)$.

4.2. Slow trend. The slowly evolving trend is assumed dependent upon both $X$ and $\tau$ at order one. Its assumed independence of the fast wave component (that appears in the full multiple-scales analysis in Section 4.3) is not general, but is convenient and correct to the order of approximation considered here.

Since $\epsilon^{2}$ appears in the dimensionless momentum/continuity equations (18) and (19), a regular perturbation is assumed for the head and velocity in even powers of the small parameter $\epsilon$. Defining the slow trend component as $H(X, \tau)=H_{0}(X, \tau)+\epsilon^{2} H_{1}(X, \tau)+\ldots$ and $V(X, \tau)=V_{0}(X, \tau)+\epsilon^{2} V_{1}(X, \tau)+\ldots$ and substituting these into (18) and (19), we find that the zeroth approximation satisfies

$$
\begin{aligned}
H_{0 X}+C_{1} V_{0} V_{0 X}+V_{0 \tau}+C_{2} V_{0}+C_{3} V_{0}^{2} & =0, \\
V_{0 X} & =0 .
\end{aligned}
$$

The second equation in (22) requires $V_{0}=V_{0}(\tau)$ while

$$
H_{0}(X)=1-X
$$

is independent of $\tau$, so as to satisfy the inhomogeneous boundary condition at $X=0$. Substitution of $V_{0}(\tau)$ to the first equation of (22) gives the first-order separable equation

$$
V_{0 \tau}=1-C_{2} V_{0}-C_{3} V_{0}^{2}
$$


with solution

$$
V_{0}(\tau)=a \tanh \left(a C_{3} \tau+D\right)-b, a=\sqrt{\frac{1}{C_{3}}+b^{2}}, b=\frac{C_{2}}{2 C_{3}},
$$

where $D$ is a free constant, the choice of which is discussed at the end of the following section. Note that this first approximation to the head and velocity is the rigid-column response to a suddenly applied pressure gradient.

4.3. Multiple scales and wave attenuation. A multiple-scales expansion is built for the head and velocity. Following the results of the previous section, the attenuated wave and trending components from the previous two subsections are summed. Although the governing equations in (18) and (19) are nonlinear, this summing is correct for small $\epsilon$ since the zeroth head trending component (23) is independent of $t$ and $\tau$ and the velocity wave component is a lower-order correction to the zeroth velocity trending component (25).

Based upon the previous section, we conjecture a complex form

$$
\begin{array}{r}
H(X, t, \tau)=P(\tau)(1+\epsilon Q(\tau)+\ldots)\left[e^{i\left(X-t / \sqrt{c_{4}}\right)}+\epsilon F_{1}\left(X-t / \sqrt{c_{4}}\right)+\ldots\right]+ \\
H_{0}(X)+\epsilon^{2} H_{1}(X)+\ldots \\
V(X, t, \tau)=\sqrt{c_{4}} \epsilon P(\tau)(1+\epsilon \tilde{Q}(\tau)+\ldots)\left[e^{i\left(X-t / \sqrt{c_{4}}\right)}+\epsilon G_{1}\left(X-t / \sqrt{c_{4}}\right)+\ldots\right]+ \\
V_{0}(\tau)+\epsilon^{2} V_{1}(X, \tau)+\ldots
\end{array}
$$

In this form, the unidirectional wave component is a complex exponential that depends upon the fast variable $t$, where the $O(\epsilon)$ components $F_{1}$ and $G_{1}$ are generically stated and may also be complex. The wave attenuation component of the head, $H$, is taken to be a multiplicative regular perturbation $P(\tau)(1+\epsilon Q(\tau)+\ldots)$ that only depends upon the slow variable $\tau$, where $P(\tau)$ conveniently represents the first attenuation approximation. The two primary assumptions in the wave component are: (i) that the head dependence on the two timescales $t$ and $\tau$ is treatly separately and (ii) that only one direction needs to be considered for the wave component since we conjecture the attenuation is independent of the wave direction and thus boundary conditions. This assumption is tested below by comparison of the analytic approximation with the numerical solution to the governing equations satisfying the boundary conditions. A similar form appears in the velocity $V$. Note that, in both cases, the second term is the trending component discussed in Section 4.2 and given in equations (23) and (25). All expansions are assumed to be in powers of $\epsilon$ as indicated by the governing equations (18) and (19).

The definition of the form in (26) and (27) leads us to the primary goal of this paper: "The discovery of the wave attenuation function $P(\tau)$ ".

Substitution of the full form to the dimensionless momentum equation in (18) yields the complex first-order equation for the wave attenuation function $P(\tau)$ as

$$
\sqrt{c_{4}} \frac{d P(\tau)}{d \tau}+\sqrt{c_{4}}\left[C_{2}+2 C_{3} V_{0}(\tau)+C_{1} V_{0}(\tau) i\right] P(\tau)+i P(\tau) \Delta Q(\tau)=0
$$




\begin{tabular}{|c|}
\hline Dimensional parameters \\
\hline Conduit diameter $D_{c}=0.3 \mathrm{~m}$ \\
Conduit length $L=1000 \mathrm{~m}$ \\
Conduit roughness $\epsilon_{c}=0.0003 \mathrm{~m}$ \\
Conduit wall thickness $w=0.005 \mathrm{~m}$ \\
Applied head change at $t=0,\left(h_{2}-h_{1}\right)=462 \mathrm{~m} ;$ see Figure 3 \\
Young's modulus of iron $E=2.0 \cdot 10^{11} \mathrm{~Pa}$ \\
Water bulk modulus $E_{v}=2.27 \cdot 10^{9} \mathrm{~Pa}$ \\
Kinematic viscosity $\nu=\mu / \rho=10^{-6} \mathrm{~m}^{2} / \mathrm{s}$ \\
\hline Derived dimensional parameters \\
Steady velocity $v_{\infty}=11.7 \mathrm{~m} / \mathrm{s}$ (25) \\
\hline Viscous constant $r=0.55 \mathrm{~s}$ \\
Inertial constant $s=3.34 \mathrm{~s}^{2} / \mathrm{m}$ \\
Dimensionless parameters \\
\hline$C_{1}=0.030, C_{2}=0.014, C_{3}=1.0$ (Momentum equation (18) $)$ \\
$C_{1} c_{4}=3.3, c_{4}=110, c_{5}=-1.9, \epsilon^{2}=10^{-3}($ Continuity equation (19) $)$ \\
\hline
\end{tabular}

TABLE 1. Parameters used for numerical simulations. Fundamental dimensional parameters are shown along with their derived dimensional and dimensionless counterparts.

where $\Delta Q(\tau)=Q(\tau)-\tilde{Q}(\tau)$. Proceeding similarly for the continuity equation in (19) gives

$$
c_{4} \frac{d P(\tau)}{d \tau}+C_{1} c_{4} V_{0}(\tau) P(\tau) i-\sqrt{c_{4}} i P(\tau) \Delta Q(\tau)=0 .
$$

Eliminating $\Delta Q(\tau)$ from these last two equations yields

$$
2 \frac{d P(\tau)}{d \tau}+\left[C_{2}+2 C_{3} V_{0}(\tau)+i\left(C_{1} V_{0}(\tau)+C_{1}\right)\right] P(\tau)=0
$$

The last equation for the attenuation can be restated as

$$
\frac{d P(\tau)}{d \tau}+\lambda(\tau) P(\tau)=0,
$$

where $\lambda(\tau)=\lambda_{R}(\tau)+i \lambda_{I}(\tau)$ is the complex term with

$$
\begin{aligned}
\lambda_{R}(\tau) & =\frac{C_{2}+2 C_{3} V_{0}(\tau)}{2}, \\
\lambda_{I}(\tau) & =\frac{C_{1} V_{0}(\tau)+C_{1}}{2},
\end{aligned}
$$

and the attenuation function is

$$
P(\tau)=\tilde{A} e^{-\int_{0}^{\tau} \lambda(z) d z},
$$

where $\tilde{A}$ is a free constant.

The magnitude of the attenuation is $|P(\tau)|=|\tilde{A}| \exp \left(\int_{0}^{\tau} \lambda_{R}(z) d z\right)$. Two free constants $\tilde{A}$ in equation (33) and $D$ in (25) remain unspecified. The free constant $D$ from (25) is chosen to satisfy the velocity initial condition and this is under the assumption 


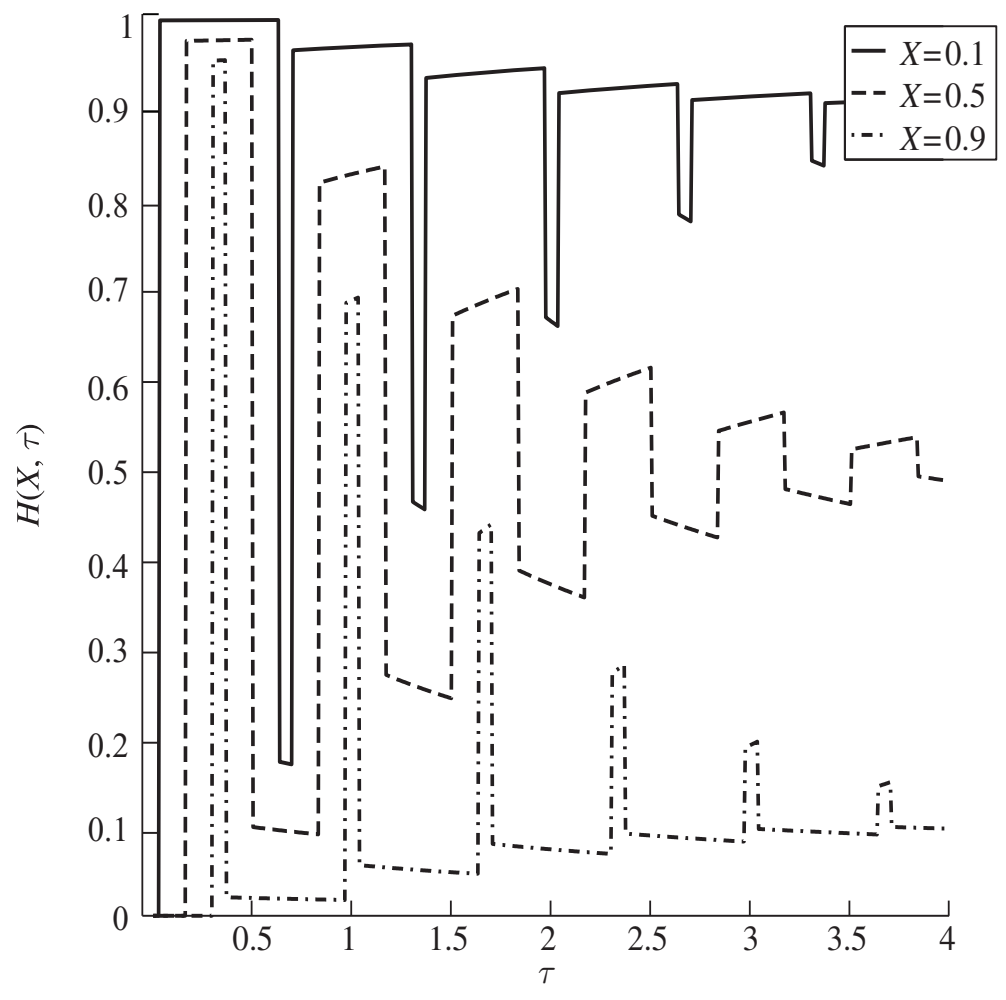

FIG. 4. The solid, dashed, and dash-dot lines are the numerical approximation to the head $H(X, \tau)$ at dimensionless positions $X=0.1$, $X=0.5$, and $X=0.9$ respectively, depicted as a function of dimensionless time $\tau$. Note that as $\tau \rightarrow \infty$ the dimensionless head is progressing to the steady state $H_{0}(X)=1-X$ (equation 231) at each $X$ location. The method of characteristics numerical solution follows the description in Chapter 4 of [18. A dimensionless spatial grid spacing of $\Delta X=0.01$ is used over $0 \leq X \leq 1$.

that the multiple-scales form satisfies the initial condition. Given a zero initial condition on velocity, we have $D=\operatorname{artanh}(b / a)$. Furthermore, since the magnitude of the oscillatory component of dimensionless head $H(x, t, \tau)$ may not exceed unity, $\tilde{A}=1 / P(0)$. Substituting the trending component $V_{0}(\tau)$ of (25) and integrating yields

$$
|P(\tau)|=\frac{\operatorname{sech}\left(a C_{3} \tau+\operatorname{artanh}(b / a)\right)}{\sqrt{1-b^{2} / a^{2}}} .
$$

Note that the simpler form $\sqrt{1-b^{2} / a^{2}}$ has replaced $\operatorname{sech}(\operatorname{artanh}(b / a))$ in the denominator. The result in equation (34) generalizes ad hoc results found in [10] in two main ways: (i) the wave attenuation function is found using a structured asymptotic approach through a direct expansion of the water hammer equations and (ii) $|P(\tau)|$ is a uniformly accurate approximation that applies for all times to the classical water hammer equations since no assumptions were made, for example, about the time dependence of the velocity 


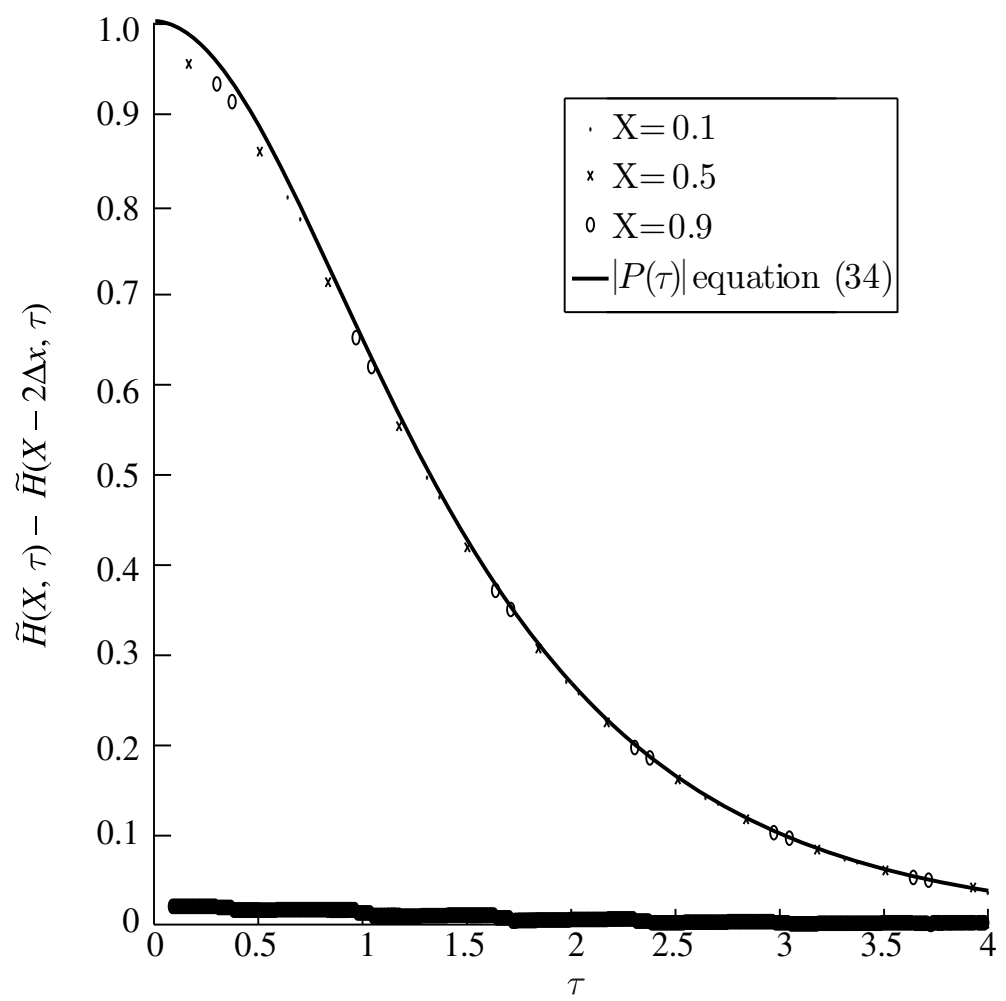

FIG. 5. The dots, crosses, and circles are the absolute difference $|\tilde{H}(X, \tau)-\tilde{H}(X-2 \Delta X, \tau)|$ where $\tilde{H}(x, \tau)=H(X, \tau)-(1-X)$ is the numerical solution in Figure 4 but with the steady-state trend $1-X$ removed. Nearly constant values of head in Figure 4 yield near zero values of absolute head difference and these are seen as a heavier line along the bottom of this figure. Values of head in Figure 4 associated with sharp changes in head in Figure 4 at each $X$ location are the remaining values and these approximate the "wave attenuation' function $|P(\tau)|$ sought after in this work. The solid line is the wave attenuation function $P(\tau)$ of equation (34) and there is close agreement with the numerical results.

$V_{0}(\tau)$. The generality of the approach and its derivation directly from the mathematical model may allow it to be used in the analysis of more difficult problems.

This first approximation to $|P(\tau)|$ is independent of $C_{1}$ (13) since that parameter is shown to influence the attenuation phase to first order, and is also independent of $c_{5}$ (14) since the pipe slope elevation head is small in relation to the celerity head from the propagating wave. Note also that, in the limit of $c_{5}$ approaching zero, the wave speed approaches infinity and rigid column motion results and the case $C_{3}=0$ corresponds to laminar flow.

The solid, dashed, and dash-dot lines in Figure 4 are the numerical approximation to the head $H(X, \tau)$ at dimensionless positions $X=0.1, X=0.5$, and $X=0.9$ respectively, 
depicted as a function of dimensionless time $\tau$. The method of characteristics numerical solution follows the description in Chapter 4 of [18. A dimensionless spatial grid spacing of $\Delta X=0.01$ is used over $0 \leq X \leq 1$. In Figure 5, the attenuation function $|P(\tau)|$ is nearly superimposed on the differenced numerical solution of Figure 4, which approximates the attenuation function as detailed in the caption.

The parameters used in the example are for a $1 \mathrm{~km}$-long water main inclined upward at an angle of $\theta=-15^{\circ}$ (see Table1). The pipe material properties and construction lead to a celerity $c_{p} \approx 1200 \mathrm{~m} / \mathrm{s}$ making the artificial parameter $\epsilon^{2}=0.001(\epsilon=0.030)$. The difference between the multiple scales and numerical results is uniformly below about 0.02 , and this size approximates $O(\epsilon)=O(0.03)$ as expected.

5. Conclusions. The method of multiple scales was used to generate a uniformly accurate approximation to the water hammer pressure-wave attenuation in the classical water hammer equations. The method was applied to water hammer initiation for a valve opening and is seen to apply to other forms of hammer initiation, including the standard valve closure or flow stoppage problem. The approach presented here is generic and should be useful for other generalizations of the classical water hammer equations to include, for example, unsteady friction.

6. Acknowledgements. The authors wish to thank the Canadian Natural Science and Engineering Research Council (NSERC) for Discovery Grant support and a reviewer for detailed comments and useful suggestions.

\section{REFERENCES}

[1] M. F. Allan and K. Al-Khaled, An approximation of the analytic solution of the shock wave equation, Journal of Computational and Applied Mathematics 192(2) (2006), 301-309. MR2228815

[2] A. S. Tijsseling and A. E. Vardy, Time scales and fsi in unsteady liquid-filled pipe flow, BHR Group, Proc. of the 9th Int. Conf. on Pressure Surges (Editor S. J. Murray), 2004, pp. 135-150.

[3] _ Time scales and fsi in oscillatory liquid-filled pipe flow, BHR Group, Proc. of the 10th Int. Conf. on Pressure Surges (Editor S. Hunt), 2008, pp. 553-568.

[4] A. Bergant, A. S. Tijsseling, J. P. Vitkovsky, D. I. C. Covas, A. R. Simpson, and M. F. Lambert, Parameters affecting water-hammer wave attenuation, shape, and timing part $i$ : Mathematical tools, IAHR J. of Hydraulic Research 46(3) (2008), 373-381.

[5] _ Parameters affecting water-hammer wave attenuation, shape, and timing part ii: Case studies, IAHR J. of Hydraulic Research 46(3) (2008), 382-391.

[6] G. C. F. M. R. de Prony Baron, Recherches physico-mathematiques sur la theorie du mouvement des eaux courantes (1804).

[7] M. S. Ghidaoui and A. A. Kolyshkin, Stability analysis of velocity profiles in water hammer flows, ASCE J. of Hydraulic Engineering 127(6) (2001), 499-511.

[8] G. O. Brown, The history of the Darcy-Weisbach equation for pipe flow resistance, Proceedings of the 150th Anniversary Conference of ASCE (Editors J. Rogers and A. Fredrich), 2002, pp. 34-43.

[9] D. Hansen, V. K. Garga, and D. R. Townsend, Selection and application of a 1-d non-Darcy flow equation for 2-d flow through rockfill embankments, Can. Geotechnical J. 32(2) (1995), 223-232.

[10] D. J. Leslie and A. S. Tijsseling, Travelling discontinuities in waterhammer theory: attenuation due to friction, BHR Group, Proc. of the 8th Int. Conf. on Pressure Surges (Editor A. Anderson), 2000, pp. 323-335.

[11] A. S. Leon, M. S. Ghidaoui, A. R. Schmidt, and M. H. Garcia, Efficient second-order-accurate shockcapturing scheme for modelling one and two-phase water hammer flows, ASCE J. of Hydraulic Engineering 134(7) (2008), 970-983. 
[12] L. F. Moody, Friction factors for pipe flow, Transactions of American Society of Mechanical Engineers 66(8) (1944), 671-678.

[13] A. M. A. Sattar, A. R. Dickerson, and M. H. Chaudhry, Wavelet-Galerkin solution to the water hammer equations, ASCE J. of Hydraulic Engineering 134(4) (2009), 283-295.

[14] A. S. Tijsseling, Fluid-structure interaction in liquid-filled pipe systems, J. of Fluids and Structures 10 (1996), 109-146.

[15] W. Driedger, Controlling centrifugal pumps, Hydrocarbon Processing 74(7) (1995), 43-49.

[16] X. Q. Wang, J. G. Sun, and W. T. Sha, Transient flows and pressure waves in pipes, Journal of Hydrodynamics 7(2) (1995), 51-59.

[17] D. H. Wilkinson and E. M. Curtis, Water hammer in a thin-walled pipe, Proc. 3rd Int. Conf. on Pressure Surges, 1980, pp. 221-240.

[18] E. B. Wylie and V. L. Streeter, Fluid transients in systems, Prentice-Hall, Englewood Cliffs, NJ, 1993. 